\title{
Nernst effect in the vortex-liquid regime of a type-II superconductor
}

\author{
Subroto Mukerje日 and David A. Hus屯 \\ Department of Physics, Princeton University, Princeton, NJ 08544
}

(Dated: June 19, 2018)

\begin{abstract}
We measure the transverse thermoelectric coefficient $\alpha_{x y}$ in simulations of type-II superconductors in the vortex liquid regime, using the time-dependent Ginzburg-Landau (TDGL) equation with thermal noise. Our results are in reasonably good quantitative agreement with experimental data on cuprate samples, suggesting that this simple model contains much of the physics behind the large Nernst effect due to superconducting fluctuations observed in these materials.

PACS numbers: 74.25.Fy, 72.15.Jf
\end{abstract}

\section{INTRODUCTION}

The Nernst effect in the cuprate superconductors has recently become a focus of attention both experimentally [1, 2, 3, 4, 5] and theoretically [6, 7, 8, 9]. The Nernst effect is the electric field induced when the sample has a temperature gradient, $\boldsymbol{\nabla} T$, perpendicular to the magnetic field, $\mathbf{H}$; this electric field is perpendicular to both $\boldsymbol{\nabla} T$ and $\mathbf{H}$. For some cuprate superconductors the Nernst effect due to superconducting fluctuations is detectable at temperatures far above the transition temperature, $T_{c}$ 3, 4]. Ussishkin et al. 7] calculated the low-field Nernst effect for $T>T_{c}$ due to superconducting fluctuations, obtaining results in reasonable agreement (in absolute units) with experimental data. They used the linearized time-dependent Ginzburg-Landau (TDGL) equation, which is identical to the Aslamazov-Larkin 10] approximation for the microscopics. Linearized (Gaussian) TDGL is applicable far enough above the mean-field transition temperature $T_{c}^{M F}$, where the order parameter fluctuations are small enough to neglect the nonlinear terms in the full Ginzburg-Landau free energy. To estimate the Nernst effect closer to $T_{c}$ they also treated the nonlinearities in TDGL in the Hartree approximation [7].

Prominent in the phase diagrams of the cuprate superconductors in a magnetic field is the vortex liquid regime, where short-range superconducting correlations are strong (because $T<T_{c}$ ), but thermal phase and vortex fluctuations disrupt the superconducting coherence on longer scales. The experimentally observed Nernst effect remains large in this regime [2, 3, 4, ,5], which is not accessible to analytic calculations due to its strong correlations and fluctuations. However, the TDGL equation with its full nonlinearity and driven by thermal fluctuations can be numerically simulated in this vortex liquid regime, and its Nernst effect measured and compared to experiments, as we demonstrate below.

When the TDGL approximation is used to model properties of cuprate superconductors, for which there is not a well-established more microscopic theory, or more gener-

\footnotetext{
*Electronic address: mukerjee@princeton.edu
}

${ }^{\dagger}$ Electronic address: huse@princeton.edu ally when it is used well away from the zero-field critical temperature, as we do here, it constitutes a standard, widely-used, but essentially phenomenological approximation. It has the virtue that most of the parameters that enter can be determined from experiments, as we discuss below. For BCS superconductors, the TDGL approximation can be systematically derived from the microscopics in some limited regimes [1, 12], but here we are instead using it more broadly, in regimes where its justification is "only" phenomenological.

The Nernst effect in the vortex liquid can be described in terms of the vortices as the phase-slip voltage due to vortices being transported down the temperature gradient as heat carriers. However, when one calculates the Nernst effect at higher temperatures in linearized TDGL [7], what is happening is that superconducting order parameter fluctuations are transported along the temperature gradient and their phase patterns are twisted by this motion across the magnetic field, inducing a Nernst voltage; vortices play no role in the calculation. In a TDGL treatment of the vortex liquid regime, there is no clean distinction between order parameter fluctuations and vortex motion, so both of these two differentsounding descriptions are in some sense correct.

In this paper, we present the method for and results of simulations of thermoelectric transport in type-II superconductors in the vortex liquid regime. We simulate the TDGL equation with thermal noise. We work in the strongly type-II limit, $\kappa \gg 1$, where the magnetic field in the sample is assumed to be uniform and not fluctuating. Mostly we work in two dimensions, but we also examine the crossover from two-dimensional to three-dimensional behavior. In our study of interlayer couplings we see some indication that a substantial part of the entropy carried by the vortices in the vortex liquid may be the configurational entropy of their positions.

Our simulations involve a dimensionless tunable parameter $(\eta)$ which sets the strength of the thermal fluctuations in the sample and hence can range from nearly mean-field behavior with very weak fluctuations to very strong fluctuations with strongly suppressed superconductivity. We show that for an intermediate value of this parameter our results are in reasonably good qualitative and quantitative agreement with available experimental Nernst-effect data on overdoped $\mathrm{La}_{2-x} \mathrm{Sr}_{x} \mathrm{CuO}_{4}$ (LSCO) 
5]. In this comparison all the other parameters in the simulations are set by experimentally measured quantities. We also discuss in brief the results of our simulations to model experiments on $\mathrm{Bi}_{2} \mathrm{Sr}_{2} \mathrm{CaCu}_{2} \mathrm{O}_{8+x}$ (BSCCO) 2].

\section{SOME THERMO-ELECTRO-MAGNETIC TRANSPORT THEORY}

We briefly review some of the general theory of thermal and electrical transport coefficients in linear response in the presence of a magnetic field. Consider a sample at temperature $T$ subjected to small gradients in the potential $\nabla \phi$ and temperature $\nabla T$. One can then very generally write down the transport current densities in the system to linear order in $\nabla \phi$ and $\nabla T$ as

$$
\begin{gathered}
\mathbf{J}_{\mathrm{tr}}^{e}=-\hat{\sigma} \nabla \phi-\hat{\alpha} \nabla T, \\
\mathbf{J}_{\mathrm{tr}}^{Q}=\hat{\tilde{\alpha}} \nabla \phi-\hat{\kappa} \nabla T,
\end{gathered}
$$

where $\mathbf{J}_{\mathrm{tr}}^{e}$ is the charge transport current density and $\mathbf{J}_{\mathrm{tr}}^{Q}$ is the heat transport current density. $\hat{\sigma}, \hat{\alpha}, \hat{\tilde{\alpha}}$ and $\hat{\kappa}$ are the electrical conductivity, thermoelectric, electrothermal and thermal conductivity tensors. The Onsager relations for the transport coefficients tell us that $\hat{\tilde{\alpha}}=T \hat{\alpha}$. The Nernst coefficent $(\nu)$ can be defined in a configuration with a magnetic field $\mathbf{B} \| \hat{\mathbf{z}}$ and $\nabla \mathbf{T} \| \hat{\mathbf{x}}$ along with the condition $\mathbf{J}_{\mathrm{tr}}^{e}=0$ as

$$
\nu=-\frac{E_{y}}{B \nabla T}=\frac{1}{B} \frac{\alpha_{x y} \sigma_{x x}-\alpha_{x x} \sigma_{x y}}{\sigma_{x x}^{2}+\sigma_{x y}^{2}} .
$$

If the system shows no Hall effect (like the one we have simulated), then $\sigma_{x y}=0$ and

$$
\nu=\frac{\alpha_{x y}}{B \sigma_{x x}} .
$$

One way to obtain transport coefficients from a simulation is via the Kubo relations, thus measuring currentcurrent correlations in the equilibrium dynamics. Alternatively, one can apply an electric field and/or a temperature gradient and measure the resulting currents. We have used both methods, finding that our implementation of the latter method gives higher signal-to-noise ratio per unit computer time in the regime we have been studying. The currents flowing in the sample have magnetization parts in addition to the transport parts considered above. One could write down linear response relations for the total current densities analogous to the ones for the transport current densities but the coefficients appearing in those would not obey the Onsager relations. The total currents in the system can in general be computed in a straightforward manner but one has to extract the transport parts from these to calculate the transport coefficients. This involves identifying and subtracting out the magnetization currents. The procedure we use to that end in this work is outlined below and is based on the arguments of Cooper et al. [13].

Let $\mathbf{J}_{\text {tot }}^{e}(\mathbf{r})$ and $\mathbf{J}_{\text {tot }}^{Q}(\mathbf{r})$ be the total charge and heat current densities (transport + magnetization) at any point $\mathbf{r}$ in the sample. If $\phi(\mathbf{r})$ is the electric potential at $\mathbf{r}$, there exists a total energy current $\mathbf{J}_{\text {tot }}^{E}(\mathbf{r})$ such that

$$
\mathbf{J}_{\text {tot }}^{Q}(\mathbf{r})=\mathbf{J}_{\text {tot }}^{E}(\mathbf{r})-\phi(\mathbf{r}) \mathbf{J}_{\text {tot }}^{e}(\mathbf{r})
$$

A similar relation holds between the transport parts of these current densities,

$$
\mathbf{J}_{\mathrm{tr}}^{Q}(\mathbf{r})=\mathbf{J}_{\mathrm{tr}}^{E}(\mathbf{r})-\phi(\mathbf{r}) \mathbf{J}_{\mathrm{tr}}^{e}(\mathbf{r})
$$

The average electric current density $\overline{\mathbf{J}}_{\text {tr }}^{e}$ and heat current density $\overline{\mathbf{J}}_{\text {tr }}^{Q}$ are given by averaging $\mathbf{J}_{\mathrm{tr}}^{e}(\mathbf{r})$ and $\mathbf{J}_{\mathrm{tr}}^{Q}(\mathbf{r})$ over the whole sample. For a two dimensional sample

$$
\overline{\mathbf{J}}_{\mathrm{tr}}^{e}=\frac{1}{S} \int_{S} \mathbf{J}_{\mathrm{tr}}^{e}(\mathbf{r}) d S
$$

and

$$
\overline{\mathbf{J}}_{\mathrm{tr}}^{Q}=\frac{1}{S} \int_{S} \mathbf{J}_{\mathrm{tr}}^{Q}(\mathbf{r}) d S
$$

where $S$ is the area of the sample. Similar relations also hold for three dimensional samples. From Eqns. (6) and (8), we obtain

$$
\overline{\mathbf{J}}_{\mathrm{tr}}^{Q}=\frac{1}{S}\left(\int_{S} \mathbf{J}_{\mathrm{tr}}^{E}(\mathbf{r}) d S-\int_{S} \phi(\mathbf{r}) \mathbf{J}_{\mathrm{tr}}^{e}(\mathbf{r}) d S\right) .
$$

We know that

$$
\begin{aligned}
& \mathbf{J}_{\text {tot }}^{e}(\mathbf{r})=\mathbf{J}_{\mathrm{tr}}^{e}(\mathbf{r})+\mathbf{J}_{\text {mag }}^{e}(\mathbf{r}) \\
& \mathbf{J}_{\text {tot }}^{E}(\mathbf{r})=\mathbf{J}_{\mathrm{tr}}^{E}(\mathbf{r})+\mathbf{J}_{\text {mag }}^{E}(\mathbf{r}),
\end{aligned}
$$

where $\mathbf{J}_{\text {mag }}^{e}(\mathbf{r})$ and $\mathbf{J}_{\text {mag }}^{E}(\mathbf{r})$ are the charge and energy magnetization current densities. It can be shown on general grounds [13] that there exist charge and energy magnetization densities $\mathbf{M}^{e}(\mathbf{r})$ and $\mathbf{M}^{E}(\mathbf{r})$ such that

$$
\begin{aligned}
& \mathbf{J}_{\text {mag }}^{e}(\mathbf{r})=\nabla \times \mathbf{M}^{e}(\mathbf{r}) \\
& \mathbf{J}_{\text {mag }}^{E}(\mathbf{r})=\nabla \times \mathbf{M}^{E}(\mathbf{r}) .
\end{aligned}
$$

The charge magnetization of the system is nothing but the conventional magnetic moment density $\mathbf{M}(\mathbf{r})$. If the material surrounding the sample is assumed to be nonmagnetic, both $\mathbf{M}^{e}(\mathbf{r})$ and $\mathbf{M}^{E}(\mathbf{r})$ vanish outside the sample. The magnetization currents are mostly at the boundaries because that is where the change in magnetization is the largest. Thus we see that

$$
\begin{aligned}
\int_{S} \mathbf{J}_{\mathrm{tr}}^{e}(\mathbf{r}) d S & =\int_{S} \mathbf{J}_{\mathrm{tot}}^{e}(\mathbf{r}) d S \\
\int_{S} \mathbf{J}_{\mathrm{tr}}^{E}(\mathbf{r}) d S & =\int_{S} \mathbf{J}_{\mathrm{tot}}^{E}(\mathbf{r}) d S .
\end{aligned}
$$


From Eqns (9) and (12), we obtain

$$
\overline{\mathbf{J}}_{\mathrm{tr}}^{Q}=\frac{1}{S}\left(\int_{S} \mathbf{J}_{\mathrm{tot}}^{E}(\mathbf{r}) d S-\int_{S} \phi(\mathbf{r}) \mathbf{J}_{\mathrm{tr}}^{e}(\mathbf{r}) d S\right) .
$$

It should be noted that there is no "heat magnetization density" $\mathbf{M}^{Q}(\mathbf{r})$ analogous to $\mathbf{M}^{e}(\mathbf{r})$ and $\mathbf{M}^{E}(\mathbf{r})$ for which the heat magnetization current $\mathbf{J}_{\text {mag }}^{Q}(\mathbf{r})=$ $\nabla \times \mathbf{M}^{Q}(\mathbf{r})$. A consequence of this is that unlike $\mathbf{J}^{e}$ and $\mathbf{J}^{E}$,

$$
\int_{S} \mathbf{J}_{\mathrm{tr}}^{Q}(\mathbf{r}) d S \neq \int_{S} \mathbf{J}_{\text {tot }}^{Q}(\mathbf{r}) d S .
$$

In fact, it can be shown that

$$
\mathbf{J}_{\operatorname{mag}}^{Q}(\mathbf{r})=\nabla \phi \times \mathbf{M}(\mathbf{r})
$$

\section{SIMULATIONS}

We measure the transport coefficient $\tilde{\alpha}_{x y}$ by turning on an electric field $-\nabla \phi \| \hat{\mathbf{y}}$ and a magnetic field $\mathbf{B} \| \hat{\mathbf{z}}$ and measuring $\bar{J}_{\mathrm{tr}}^{Q(x)}$, the $x$ component of the average heat transport current density at constant temperature. This is done in a system with mixed boundary conditions: it has free surfaces at the boundaries normal to $\hat{y}$, while it has periodic boundary conditions along the $x$-direction (and along $\hat{z}$ when we study 3D). If, as in our model, the system does not have a Hall response, under these conditions the $x$ component of the electric transport current vanishes at all points, $J_{\mathrm{tr}}^{e(x)}(\mathbf{r})=0$. Thus from Eqns. (2) and (13), we obtain

$$
\tilde{\alpha}_{x y}=-\frac{\bar{J}_{\mathrm{tr}}^{Q(x)}}{\nabla_{y} \phi}=-\frac{1}{S} \frac{\left(\int_{S} J_{\mathrm{tot}}^{E(x)}(\mathbf{r}) d S\right)}{\nabla_{y} \phi} .
$$

Similarly, $\alpha_{x y}$ can be measured by introducing a temperature gradient $\nabla T \| \hat{\mathbf{y}}$ and magnetic field $\mathbf{B} \| \hat{\mathbf{z}}$ with no external electric potential and measuring $\bar{J}_{\mathrm{tr}}^{e(x)}$. From Eqns. (1) and (12),

$$
\alpha_{x y}=-\frac{\bar{J}_{\mathrm{tr}}^{e(x)}}{\nabla_{y} T}=-\frac{1}{S} \frac{\left(\int_{S} J_{\mathrm{tot}}^{e(x)}(\mathbf{r}) d S\right)}{\nabla T} .
$$

The two coefficients $\tilde{\alpha}_{x y}$ are $\alpha_{x y}$ can thus be obtained by measuring only the total currents in the system. They are related to each other by the Onsager relation and to the Nernst coefficient by Eqn. (4).

The TDGL equation that we have simulated is

$$
\begin{aligned}
\tau\left(\partial_{t}+i \frac{e^{*}}{\hbar} \phi\right) \Psi= & \frac{\hbar^{2}}{2 m^{*}}\left(\boldsymbol{\nabla}-i \frac{e^{*}}{\hbar} \mathbf{A}\right)^{2} \Psi \\
& -a_{0}\left(T-T_{c}^{M F}\right) \Psi-b|\Psi|^{2} \Psi+\zeta(\mathbf{r}, t)
\end{aligned}
$$

We take $\tau$ to be real so there is neither Hall effect nor Seebeck effect [6]. This approximation of leaving out the
Hall effect should be reasonable as long as the Hall angle is small, as it generally is in the vortex liquid regime of the cuprate superconductors. We work in the type-II limit where the magnetic field is assumed to be uniform and not fluctuating. The noise correlator is

$$
<\zeta^{*}\left(\mathbf{r}^{\prime}, t^{\prime}\right) \zeta(\mathbf{r}, t)>=2 \tau k_{B} T \delta\left(\mathbf{r}-\mathbf{r}^{\prime}\right) \delta\left(t-t^{\prime}\right) .
$$

The current operators we need are [6]

$$
\begin{aligned}
& \mathbf{J}_{\text {tot }}^{e}=-i \frac{e^{*} \hbar}{2 m^{*}}\left\langle\Psi^{*}\left(\nabla-\frac{i e^{*}}{\hbar} \mathbf{A}\right) \Psi\right\rangle+c . c . \\
& \mathbf{J}_{\text {tot }}^{E}=-\frac{\hbar^{2}}{2 m^{*}}\left\langle\frac{\partial \Psi^{*}}{\partial t}\left(\nabla-\frac{i e^{*}}{\hbar} \mathbf{A}\right) \Psi\right\rangle+\text { c.c. }
\end{aligned}
$$

The TDGL equation is the simplest dynamical stochastic equation one can obtain from the Ginzburg-Landau free energy functional. As a consequence of its simple relaxational dynamics, it does not explicitly conserve either total charge or total energy. It is implicitly in contact with and exchanging energy and charge with local reservoirs. In reality these reservoirs are presumably the phonons, quasiparticles and other excitations not included in the TDGL equation. Nevertheless, the charge and energy currents carried by the superconducting order parameter $\Psi$ and its fluctuations are as given above and can be measured and their transport properties determined within this model. Charge and energy conservation can explicitly be taken into account in microscopic derivations of the TDGL theory, where only the contribution due to superconducting fluctuations is retained. It can however be shown that other effects (normal state contributions etc.) do not contribute as significantly to the Nernst effect as superconducting fluctuations [8].

An important feature of TDGL for the present work is that the parameter $\tau$, which sets the time scale, does not enter in the values of the transverse thermoelectric coefficients that we are studying, $\alpha_{x y}$ and $\tilde{\alpha}_{x y}$. This allows quantitative comparison to experiments to be done without estimating $\tau$, which is fortunate, because the value of $\tau$ is quite uncertain.

This TDGL equation with noise has a dimensionless parameter that gives the strength of the thermal fluctuations. To remove all the dimensional quantities, we use $\xi_{0}$, the zero temperature coherence length, as our unit of length; $T_{c}^{M F}, k_{B} T_{c}^{M F}, \frac{\hbar}{e^{*}}$ and $\tau /\left(a_{0} T_{c}^{M F}\right)$ as our units of temperature, energy, magnetic flux and time, respectively; and $\Psi_{0}$, the zero-temperature order parameter magnitude, as our unit for the order parameter. The resulting TDGL equation is then

$$
\left(\partial_{t}+i \phi\right) \Psi=(\boldsymbol{\nabla}-i \mathbf{A})^{2} \Psi-(T-1) \Psi-|\Psi|^{2} \Psi+\zeta(\mathbf{r}, t),
$$

with noise correlator

$$
<\zeta^{*}\left(\mathbf{r}^{\prime}, t^{\prime}\right) \zeta(\mathbf{r}, t)>=2 \eta T \delta\left(\mathbf{r}-\mathbf{r}^{\prime}\right) \delta\left(t-t^{\prime}\right),
$$

and

$$
\eta=\frac{b k_{B} T_{c}^{M F}}{\xi_{0}^{d}\left(a_{0} T_{c}^{M F}\right)^{2}}
$$


is the fluctuation parameter, where $d$ is the number of spatial dimensions. When $\eta \ll 1$, the actual critical temperature $T_{c}$ is near the mean-field critical temperature $T_{c}^{M F}$, while if $\eta \gg 1$ then $T_{c} \ll T_{c}^{M F}$. The model has four parameters that remain after scaling to the units specified above: the temperature, the magnetic field, the cutoff, and the strength $\eta$ of the noise.

We initially simulate a two-dimensional system, thus ignoring interlayer coupling. We discretize space and time. The spatial grid spacing is taken to be $\xi_{0}$. This rather coarse spatial grid is used to minimize the computer time needed to simulate samples large compared to this microscopic length. There are noticeable quantitative effects of using such a coarse grid. For example, in our units $H_{c 2}(T=0) \cong 1.18$ with this grid, while it is 1.0 for the continuum model. However, recognizing that the model we are simulating is rather simple, so should not be expected to give highly precise quantitative results, our goal in the present study is to be quantitatively only as accurate as might be expected of such a simple model. Although we do not know how accurate that really is, the general precision to which we have chosen to work is roughly $10 \%$ to $20 \%$. The quantitative shifts due to our using a coarse grid in the simulations are at most of this magnitude. We have also verified that using a finer grid spacing would not alter our results in any significant way. Reduction of the grid spacing adds more degrees of freedom, resulting in increased fluctuations, and suppression of $T_{c}$ and $H_{c 2}$. However, if one also lowers the value of the fluctuation parameter $\eta$ a little, this increase in the fluctuations can be removed, and the resulting behavior of the system is only weakly dependent on the grid spacing.

In the simulations, the time step is chosen to be between 0.02 and 0.1 in our units, with shorter steps at higher temperatures; we check that our results are not affected by doubling the time step. A first-order Euler method is used, with 10000 to 20000 time steps used for equilibration, and 30000 to 50000 steps for averaging. The two-dimensional data we show are for a $50 \times 50$ square grid. We use the gauge-invariance of the TDGL equation to work as much as possible in terms of gauge-invariant quantities like $|\Psi|$ and the gaugeinvariant phase differences

$$
\omega\left(\mathbf{r}, \mathbf{r}^{\prime}, t\right)=\theta\left(\mathbf{r}^{\prime}, t\right)-\theta(\mathbf{r}, t)-\int_{\mathbf{r}}^{\mathbf{r}^{\prime}} \mathbf{A} \cdot \mathbf{d} \mathbf{l},
$$

where $\Psi=|\Psi| e^{i \theta}$. The current densities are also written in terms of these quantities.

At $H=0$, for the two-dimensional system there is a Kosterlitz-Thouless 14 transition at $T_{c}$, which we locate by measuring the helicity modulus.

To obtain $\tilde{\alpha}_{x y}$, we measure the transverse energy current due an electric field. We then use the Onsager relation to obtain $\alpha_{x y}=\tilde{\alpha}_{x y} / T$. We find that the signal to noise ratio for this measurement is significantly smaller than in a direct measurement of $\alpha_{x y}$. We have numerically verified the validity of this Onsager relation by measuring $\alpha_{x y}$ both ways. This also serves as a check that we are indeed measuring the proper transport coefficients. We also confirm that our results agree with the analytic results [] in the higher-temperature regime where linearized TDGL applies.

The temperature enters the TDGL equation both in the term linear in the order parameter and in the intensity of the noise. It can be shown that introducing a gradient in the linear term only adds to the magnetization currents, and thus for measuring the transport currents it is sufficient to include the temperature gradient only in the noise term. We have also verified this numerically.

\section{RESULTS}

Some results for $\alpha_{x y}$ from the two-dimensional simulations are shown in Fig. 1. We have chosen to compare these results to the experimental results of Wang et al. [5] obtained from Nernst and resistivity measurements on an overdoped LSCO sample with $x \cong 0.2$ and $T_{c} \cong 28 \mathrm{~K}$. For this comparison, we present the simulation results in SI units using the parameters from the experiment, $H_{c 2}(T=0) \cong 45 \mathrm{~T}$ (thus $\xi \cong 27 \AA$ ) and layer spacing $s=6.6 \AA$. The fluctuation parameter has been adjusted to $\eta=0.42$ to give reasonable quantitative agreement between simulation and experiment. We set the temperature scale using $T_{c}^{M F}=40 \mathrm{~K}$, which gives $T_{c} \cong 28 \mathrm{~K}$ in the simulation. Note that $\alpha_{x y}$ is not directly measured in the experiments. For a system with negligible Hall effect, $\alpha_{x y}$ can be obtained from the measured Nernst effect and the longitudinal resistivity. Thus for this comparison we require both these measurements to be made on the same sample over a substantial portion of the vortex liquid regime. This requirement seriously limits the number of experimental results we can compare to.

Looking at Fig. 1, we see that there is reasonably good general qualitative and quantitative correspondence between the simulation and experiment, considering how simple the model is and that we have adjusted only the strength of the thermal fluctuations $\eta$ to get this agreement. The other parameters are all dictated by experiment. Our results are thus consistent with the proposition that the superconducting fluctuations modelled by the TDGL equation produce most, if not all, of the contributions to the large Nernst effect seen in the vortex liquid regime for this cuprate sample. Of course, the agreement between experiment and this simple model is not perfect. One difference is seen at high field in the higher- $T$ panel of Fig. 1, where the simulations give a nearly-constant $\alpha_{x y}$, while the experiment shows more variation.

One effect that is not included in our two-dimensional simulations is interlayer coupling. For the cuprates, this can become important in the vortex liquid at low fields in the vicinity of $T_{c}$, where there is a crossover to threedimensional behavior as the correlation length normal to the layers becomes larger than one layer spacing. 

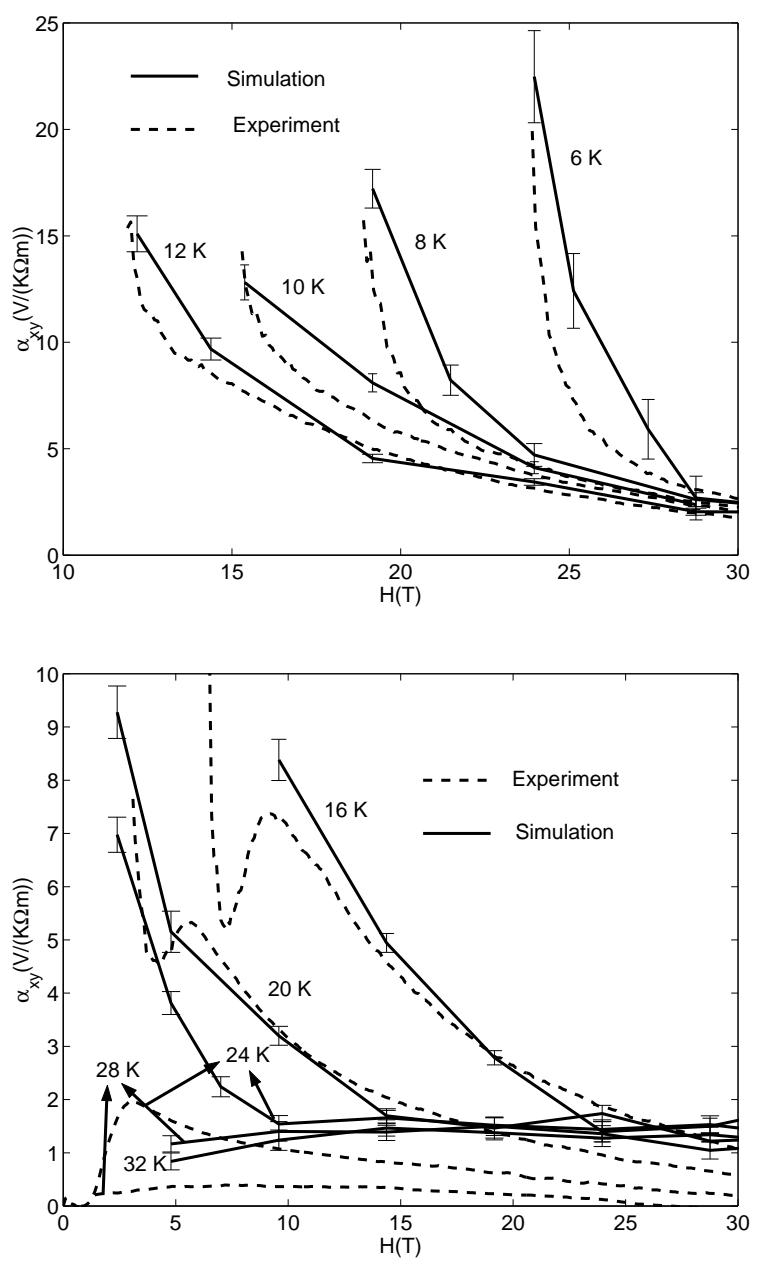

FIG. 1: Experimental data obtained from measurements [5] of the Nernst effect on overdoped LSCO $(x=0.2)$ along with the results of our two-dimensional simulations. The parameters used in the simulation are: $T_{c}^{M F}=40 \mathrm{~K}, H_{c 2}(0)=45 \mathrm{~T}, s=$ $6.6 \AA, \eta=0.42$. The critical temperature is $T_{c} \approx 28 \mathrm{~K}$ in both simulation and experiment here.
Note that the difference between simulation and experiment is large at low fields and $T=24 \mathrm{~K}$. We considered the possibility that this difference might be due to the crossover to three-dimensional behavior, which is not present in our two-dimensional simulation. To investigate this crossover, we have also simulated the LawrenceDoniach [15] version of the TDGL equation. In our units this is

$$
\left(\partial_{t}+i \phi\right) \Psi_{j}=\left(\nabla_{\perp}-i \mathbf{A}_{\perp}\right)^{2} \Psi_{j}-(T-1) \Psi_{j}+J\left(e^{-i s A_{z}} \Psi_{j+1}+e^{i s A_{z}} \Psi_{j-1}-2 \Psi_{j}\right)+\left|\Psi_{j}\right|^{2} \Psi_{j}+\zeta
$$

where $J$ is the interlayer Josephson coupling per unit area, and $j$ is the layer index. The transition temperature is located by finding the intersections of the fourth-order cumulant (Binder ratio) curves $\left\langle|\psi|^{4}\right\rangle /\left\langle|\psi|^{2}\right\rangle^{2}$ for different system sizes as functions of temperature at zero magnetic field.

Fig. 2 shows results for $\alpha_{x y}$ as a function of field for various values of interlayer coupling $J$ at temperature $T=24 \mathrm{~K}$, from samples of size $15 \times 15 \times 10$ layers. For each $J$ we set the the temperature scale $\left(T_{c}^{M F}\right)$ so that $T_{c} \cong 28 \mathrm{~K}$ as in the experiment. The values of the other parameters in the simulations are as used in Fig. 1. As expected, the the largest value of $J$ shows the largest deviation from the two-dimensional behavior. Somewhat better correspondence between the behavior of $\alpha_{x y}$ obtained in simulation and experiment is obtained by adding this interlayer coupling.

An interesting point to note in Fig. 2 is that at low fields a rather small interlayer coupling $J$ produces a strong decrease in $\alpha_{x y}$. Describing this in terms of vortices, as seems appropriate in this low-field regime below $T_{c}$, the force per unit length on a vortex line due to the 


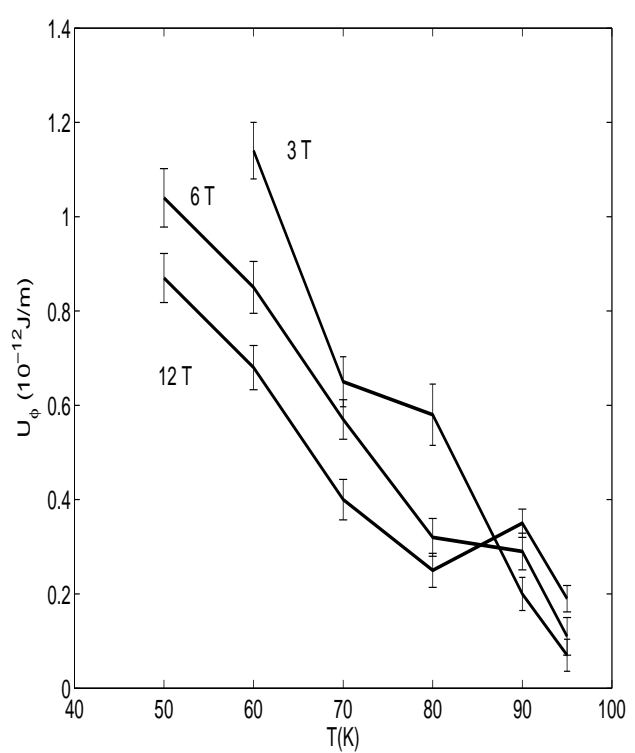

FIG. 3: (Left) Data from the simulation with $s=15.35 \AA$, $T_{c}=90 \mathrm{~K}, H_{c 2}=160 \mathrm{~T}$ and $\eta=0.475$. (Right) Experimentally measured [2] value of $U_{\phi}$ obtained from Nernst measurements on optimally doped BSSCO (2212) with $T_{c}=90 \mathrm{~K}$ and $H_{c 2}(0) \approx 160 \mathrm{~T}$.



FIG. 2: Three-dimensional simulation results for $T=24 \mathrm{~K}$, with interlayer coupling $J$. Except for $T_{c}^{M F}$, the parameters used here are the same as in Fig. 1. See text for more details.

temperature gradient is proportional to $\alpha_{x y}$. Naively, this force is also set by the entropy per unit length transported by a moving vortex. This transport entropy may come from internal degrees of freedom within one vortex, or it may come from the configurational entropy of

where the sign of the temperature derivative of $U_{\phi}$ ap- the many possible spatial arrangements of the vortices. The latter does not involve internal excitations within the vortices. In the quasi-two-dimensional layered superconductors that we are modelling, a vortex line running normal to the layers consists of "pancake vortices" in each layer. A weak interlayer coupling has little effect on the internal properties of a single pancake vortex, but it does produce an attraction between vortices in adjacent layers that reduces their relative motion, and thus can substantially reduce the configurational entropy of the vortices. The fact that at low fields a weak interlayer coupling greatly reduces the transport entropy of the vortices, as indicated by the reduction of $\alpha_{x y}$ seen in Fig.2, thus suggests that in this regime the configurational entropy of the vortices is a large part of their transport entropy, at least within this layered TDGL model.

We have also performed simulations to model the BSCCO sample studied by Ri et al. [2]. The experimental data and the results of the simulation are shown in Fig. 3. The quantity plotted is the measured "transport energy" of the vortices, defined as $U_{\phi}=T \phi_{0} \alpha_{x y}$. We used a two-dimensional simulation with $T_{c} \cong 90 \mathrm{~K}$, $H_{c 2}=160 \mathrm{~T}, s=15.35 \AA$ and $\eta=0.475$. Again, there is reasonable quantitative agreement, in absolute units, between the experimental data and the simulations. The clearest deviation here occurs for $T<60 \mathrm{~K}$ and $H>6 \mathrm{~T}$,

pears to differ between simulation and experiment. 
In conclusion, we have measured the transverse thermoelectric transport coefficient $\alpha_{x y}$ in simulations of the vortex-liquid regime of superconductors modeled by the TDGL equation with thermal fluctuations. We find that our simulations of a two-dimensional superconductor reproduce reasonably well much of the qualitative and quantitative features of available experimental data from some cuprate high-temperature superconductors. We have also studied the crossover from two-dimensional to three-dimensional behavior in a layered superconductor. This crossover study indicates that the configurational entropy of the vortices may constitute a large part of their transport entropy at low magnetic field just below $T_{c}$.

\section{Acknowledgments}

We thank Yayu Wang and N. P. Ong for providing us with the LSCO data. We also thank them, Shivaji Sondhi and especially Iddo Ussishkin for many useful discussions. This work is supported by the NSF through MRSEC grant DMR-0213706.
[1] T. T. M. Palstra, B. Batlogg, L. F. Schneemeyer and J. V. Waszczak, Phys. Rev. Lett. 64, 3090 (1990).

[2] H. C. Ri, R. Gross, F. Gollnik, A. Beck, R. P. Huebner, P. Wagner, and H. Adrian, Phys. Rev. B. 50, 3312 (1994).

[3] Z. A. Xu, N. P. Ong, Y. Wang, T. Kakeshita, and S. Uchida, Nature 406, 486 (2000).

[4] Y. Wang, Z. A. Xu, T. Kakeshita, S. Uchida, S. Ono, Y. Ando, and N. P. Ong, Phys. Rev. B 64, 224519 (2001).

[5] Y. Wang, N. P. Ong, Z. A. Xu, T. Kakeshita, S. Uchida, D. A. Bonn, R. Liang, and W. N. Hardy, Phys. Rev. Lett. 88, 257003 (2002).

[6] S. Ullah and A. T. Dorsey, Phys. Rev. B 44, 262 (1991).

[7] I. Ussishkin, S. L. Sondhi, and D. A. Huse, Phys. Rev. Lett 89, 287001 (2002).

[8] I. Ussishkin, Phys. Rev. B 68, 024517 (2003).

[9] S. Tan and K. Levin, cond-mat/0302248.

[10] L. G. Aslamazov and A. I. Larkin (1968), [Sov. Phys.
Solid State 10875 (1968)].

[11] M. Tinkham, Introduction to Superconductivity, (McGraw-Hill, New York, 1996), and references therein.

[12] A. I. Larkin and A. A. Varlamov, in Handbook on Superconductivity, Vol 1: Conventional and High- $T_{c}$ Superconductors, edited by K. H. Bennemann and J. B. Ketterson, (Springer, Berlin, 2003).

[13] N. R. Cooper, B. I. Halperin, and I. M. Ruzin, Phys. Rev. B 55, 2344 (1997).

[14] J. M. Kosterlitz and D. J. Thouless, J. Phys. C 6, 1181 (1973).

[15] W. E. Lawrence and S. Doniach, Proc. 12th Int. Conf. Low Temp. Phys., E. Kanda, ed. (Kyoto 1970, Keigaku Publ. Co. 1971), p. 361. 